Research Paper

\title{
Prophylactic Endoscopic Therapy for Variceal Bleeding in Patients with Hepatocellular Carcinoma
}

\author{
Jie Chen ${ }^{1,2^{*}}$, Yujen Tseng $3^{*}$, Tiancheng Luo ${ }^{1,2^{*}}, \mathrm{Na} \mathrm{Li}^{4}$, Lili Ma5 ${ }^{5}$, Shiyao Chen ${ }^{1,2,5 \bowtie}$ \\ 1. Department of Gastroenterology and Hepatology, Zhongshan Hospital, Fudan University, Shanghai, China \\ 2. Shanghai Institute of Liver Disease, Shanghai, China \\ 3. Department of Digestive diseases, Huashan Hospital, Fudan University, Shanghai, China \\ 4. Department of Infectious diseases, Zhongshan Hospital, Fudan University, Shanghai, China \\ 5. Endoscopy Center and Endoscopy Research Institute, Fudan University, Shanghai, China \\ * Authors contributed equally and share first authorship
}

$\square$ Corresponding author: Shi-yao Chen, MD, PhD, Department of Gastroenterology and Hepatology, Endoscopy Center and Endoscopy Research Institute, Zhongshan Hospital, Fudan University; Shanghai Institute of Liver Disease, Shanghai, China, 180 Fenglin Road, Shanghai, China, 0086200032. Tel: +8613601767310, +8613764633539; Fax: 862164038472; Email: chen.shiyao@zs-hospital.sh.cn.

(C) Ivyspring International Publisher. This is an open access article distributed under the terms of the Creative Commons Attribution (CC BY-NC) license (https://creativecommons.org/licenses/by-nc/4.0/). See http://ivyspring.com/terms for full terms and conditions.

Received: 2018.10.05; Accepted: 2019.04.13; Published: 2019.06.02

\begin{abstract}
Background \& Aims: To evaluate the efficacy and timing of secondary prophylactic endoscopic therapy for variceal bleeding. To determine the long-term survival and identify the prognostic factors in patients with hepatocellular carcinoma.

Methods: A Retrospective study was conducted. We included patients with concurrent hepatic carcinoma who received endoscopic therapy for variceal bleeding. A Kaplan-Meier survival analysis was performed. Potential factors for prediction were compared with Cox's proportional hazard model analysis.

Results: 192 patients were included between January 2008 and December 2014. During follow-up (median 17 months), 102 patients presented with an episode of rebleeding. The cumulative 6-month, 1- and 5-year rebleeding rates were $40.9 \%, 49.3 \%$ and $71.2 \%$. A total of 98 patients died during follow-up. The overall 6-month, 1 -and 5 -year cumulative mortality rates were $33.5 \%, 45.8 \%$ and $65.7 \%$. BCLC C/D stage ( $P=0.035$, $\mathrm{HR}=1.53,95 \% \mathrm{Cl} 1.003-2.327$ ) was an independent predictor of bleeding relapse, while BCLC C/D staging $(\mathrm{P}=0.035, \mathrm{HR}=1.59,95 \% \mathrm{Cl} 1.033-2.454)$ and male gender $(\mathrm{P}=0.007, \mathrm{HR}=1.58,95 \% \mathrm{Cl} 1.135-2.198)$ predicted shorter survival times. Combination ligation and cyanoacrylate was associated with a decreased rebleeding and mortality rate in patients with both esophageal and gastric varices $(P=0.003)$. The start time of the initial endoscopic procedure did not affect treatment efficacy.

Conclusions: Ligation combined with cyanoacrylate could be preferred for HCC patients presented with both esophageal and gastric varices. BCLC C/D stage predicted shorter survival times and bleeding relapse, while male gender was an independent predictor of poor survival.
\end{abstract}

Key words: Hepatocellular carcinoma, variceal bleeding, endoscopic therapy, liver cirrhosis

\section{Introduction}

Esophagogastric variceal bleeding represents a common complication in patients with hepatocellular carcinoma (HCC). Tumor infiltration could aggravate portal hypertension, resulting in the development of collateral circulation and esophagogastric varices (EGV). A previous study showed that $63.3 \%$ of the HCC patients have concomitant varices, associated with a higher risk of death from bleeding 1, 2. The presence of EGV was an independent determinant of the patient prognosis. Thus, the natural history and prognosis of HCC patients with EGV are different from those without, but few studies have focused on this specific group ${ }^{3}$.

Patients with HCC are seldom cured and have limited survival. Bleeding from EGV in these patients is a lethal complication, which might become a terminal event if left untreated or no further prophylactic measure is taken. Patients with advanced HCC often present with portal thrombosis, metastasis or poor liver function, making surgery or 
transjugular intrahepatic portosystemic shunts (TIPS) difficult and inappropriate. Propranolol might negatively impact survival in patients with cirrhosis and refractory ascites ${ }^{4}$. In many cases, endoscopic therapy is the only feasible therapeutic option. In addition, maintenance ligation lowers the recurrent bleeding rate if patients have an adequate hepatic reserve ${ }^{5}$.

The purpose of this study was to evaluate the efficacy and timing of endoscopic therapy for EGV bleeding in patients with HCC and identify the potential prognostic factors for long-term survival in these patients.

\section{Patients and Methods}

A retrospective study was conducted. Patients admitted to our hospital between January 2008 and December 2014 for acute variceal bleeding and previously diagnosed with HCC were included. Exclusion criteria were: (1) No history of variceal bleeding. (2) Previous endoscopic treatments for EGV before the occurrence of HCC (3) Co-morbid diseases that greatly compromised survival like uremia or malignancy other than hepatocellular carcinoma. The protocol of the study was approved by Ethics Committee of Zhongshan Hospital, Fudan University (approval No.: B2012-028, date of review: May 22, 2012) and conforms to the provisions of the Declaration of Helsinki.

The diagnosis of HCC was based on the histopathological data obtained from surgical resection or persistent elevated serum a-fetoprotein (AFP) levels and characteristic imaging findings (CT, MRI and/or hepatic angiography). We used the Barcelona Clinic Liver Cancer(BCLC) grading system ${ }^{6}$ to further classify the patients. Cirrhosis was histologically determined in combination with the results of laboratory and radiological analysis. The Child-Pugh score ${ }^{7}$ as calculated for each patient to evaluate liver function. The laboratory results were obtained within 3 days before the first endoscopic treatment.

All patients enrolled in this study were followed up until death or December 31, 2014 and monitored for rebleeding, survival and any causes of death.

\section{Treatment for HCC}

The treatment strategy for HCC was dependent on the individual disease state and preference, including hepatic resection, transcatheter arterial chemoembolization (TACE), radiofrequency ablation (RFA), radiotherapy, percutaneous ethanol injection (PEI), chemotherapy, palliative treatment or a combination of any of these therapies.

\section{Management of acute EGV bleeding}

All patients were administered topical anesthesia with $2 \%$ lidocaine and premedicated with an intravenous injection of propofol $(10 \mathrm{ml})$. An electronic endoscope GIF-XQ240/H260 (Olympus, Tokyo, Japan) was used for screening and treatment. Esophageal varices (EV) were categorized according to the classification of the Chinese Society of Gastroenterology and Chinese Society of Endoscopy, 8 where Mild (G1) varices are denoted by linear or slightly tortuous, red color sign(-); Moderate (G2) varices are denoted by either1) linear or slightly tortuous with red color $\operatorname{sign}(+)$ or 2) serpentine-like dilation with red color sign(-); and Severe (G3) varices are denoted by either1) serpentine-like dilation with red color sign(+) or2) pearl, modular or tumor-like dilatation. The location of the gastric varices(GV) was classified according to Sarin 9, 10 as lesser curvature varices(GOV-1), greater curvature varices(GOV-2) or both (GOV-3).

Endoscopic injection sclerotherapy (EIS): The intravariceal or paravariceal injection of 10 to $30 \mathrm{ml}$ of lauromacrogol (Tianyu Pharmaceutical, China) per session was administered through the injection needle (Olympus NM-200L-423, Japan). The initial injections were administered at 2-3 cm above the gastroesophageal junction.

Endoscopic variceal ligation (EVL): Variceal ligation was performed using 6 multi-band ligators (Cook Endoscopy, Winston-Salem, NC) or 7 multi-band ligators (Boston Scientific Corp, Natick, Mass). The ligations were initiated at $1 \mathrm{~cm}$ above the gastroesophageal junction and proceed to the next proximal varix.

Endoscopic injection of tissue adhesive (N-butyl-cyanoacrylate, NBCA): After flushing the needles with isotonic sodium chloride solution, the intravariceal injection of NBCA (Beijing Suncon Medical Adhesive Co., Ltd)was performed to target the GVs. Each injection contained different quantities of NBCA (0.5-2.0 cc), as needed, and an equal volume of lipiodol or $20 \%$ glucose.

All patients presenting with active GI bleeding received continuous intravenous infusion of vasoactive drugs before endoscopic therapy and maintained for 5 days or at least 24h without signs of re-bleeding. Follow-up endoscopy was performed at 6-8-week intervals, unless the patient missed an appointment, and therapy was repeated when necessary until complete obliteration was achieved.

\section{Statistical analysis}

Statistical analysis was performed using SPSS 16.0 for Windows. Kaplan-Meier survival analysis was conducted to estimate cumulative overall 
survival, and the log-rank test was used to compare survival distributions. Potential prognostic factors were identified using Cox's proportional hazard model analysis. All variables with a univariate $P$ value of $<0.05$ were included in the multivariate analysis. $\mathrm{P}<0.05$ was considered significant.

\section{Results}

\section{Patient characteristics}

From January 2008 to December 2014, a total of 192 patients (median age: 55 years, range 25-81 years) met the criteria for inclusion in this study. The study flow diagram was shown in Figure 1. The baseline characteristics of the patients were shown in Table 1. The median follow-up period of all patients was 17 (range 0.1- 72.7) months.7 patients (3.6\%) were lost to follow up. Endoscopic treatment was performed with an average of 1.5 sessions. The patients were classified using the Child-Pugh score as Child A in 32 cases (16.7\%), Child B in 158 cases (82.3\%) and Child C in 2 cases $(1.0 \%)$. According to BCLC grading system, there were 77 BCLC-A patients (40.1\%), 56 BCLC-B patients $(29.2 \%), 57$ BCLC-C patients $(29.7 \%)$, and 2 BCLC-D patients $(1.0 \%)$.

\section{Re-bleeding analysis}

During the follow-up period, 102 patients $(51.7 \%)$ presented with an episode of rebleeding. The cumulative 6-month, 1- and 5-year rebleeding rates were $40.9 \%, 49.3 \%$ and $71.2 \%$, respectively (Figure 2A). 16 patients presented with failure to control acute bleeding, which was defined as bleeding relapse within 5 days after the initial endoscopic procedure, while primary hemostasis was achieved in $91.7 \%$ of the patients. We investigated the potential risk factors that could influence prognosis. Table 2 summarized the results of the Cox multivariate analyses. The results indicated that different endoscopic procedures did not influence the risk of rebleeding (EVL vs non-EVL, $\mathrm{P}=0.572)$. However, BCLC C/D stage $(\mathrm{P}=0.048, \quad \mathrm{HR}=1.53, \quad 95 \% \quad \mathrm{CI} 1.003-2.327)$ was independent predictors for the relapse of bleeding in these patients (Figure 2B).

Table 1. Baseline characteristics of the patients

\begin{tabular}{ll}
\hline Characteristics & Patients(n=192) \\
\hline Gender(male/ female) & $161(83.9 \%) / 31(16.1 \%)$ \\
Age(years old) & $55(25-81)$ \\
Child-Pugh score (A/B/C) & $32(16.7 \%) / 158(82.3 \%) / 2(1.0 \%)$ \\
Meld score & $10.2 \pm 2.6$ \\
BCLC grading (A/B/C/D) & $77(40.1 \%) / 56(29.2 \%) / 57(29.7 \%) / 2(1.0 \%)$ \\
Treatment for HCC (Surgical & $42(21.9 \%) / 1(0.5 \%) / 72(37.5) /$ \\
resection/Transplantation/TACE & $47(24.5) / 27(14.1 \%) / 3(1.6 \%)$ \\
/ Combination/Palliative/ & \\
Uncertain) & $171(89.1 \%) / 4(2.1 \%) / 2(1.0 \%) / 4(2.1 \%) / 11(5.7 \%$ \\
Cause of cirrhosis(HBV/ HCV/ & \\
Alcoholic/ & \\
schistosomiasis/others) & $23(12.0 \%) / 169(88.0 \%)$ \\
Propranolol treatment(yes/no) & $20(10.4 \%) / 172(89.6 \%)$ \\
Splenectomy(yes/no) & $27(14.1 \%) / 165(85.9 \%)$ \\
Diabetes mellitus(yes/no) & $4(2.1 \%) / 6(3.1 \%) / 16(8.3 \%) / 166(86.5 \%)$ \\
Esophageal & \\
varices(None/GI/GII/GIII) & $185(98.4 \%) / 3(1.6 \%)$ \\
Red color sign(yes/no) & $91(47.4 \%) / 101(52.6 \%)$ \\
Gastric varices(yes/no) & $68(74.7 \%) / 22(24.2 \%) / 1(1.1 \%)$ \\
GOV typing(1/2/3) & $161(83.9 \%) / 31(16.1 \%)$ \\
Portal hypertensive & \\
gastropathy(yes/no) & \\
\hline † BCLC: the Barcelona Clinic Liver Cancer grading system, TACE: transcatheter \\
arterial chemoembolization, RFA: radiofrequency ablation, PEI: percutaneous \\
ethanol injection, HBV: hepatic B virus, HCV: hepatic C virus, GOV: \\
gastroesophageal varices.
\end{tabular}

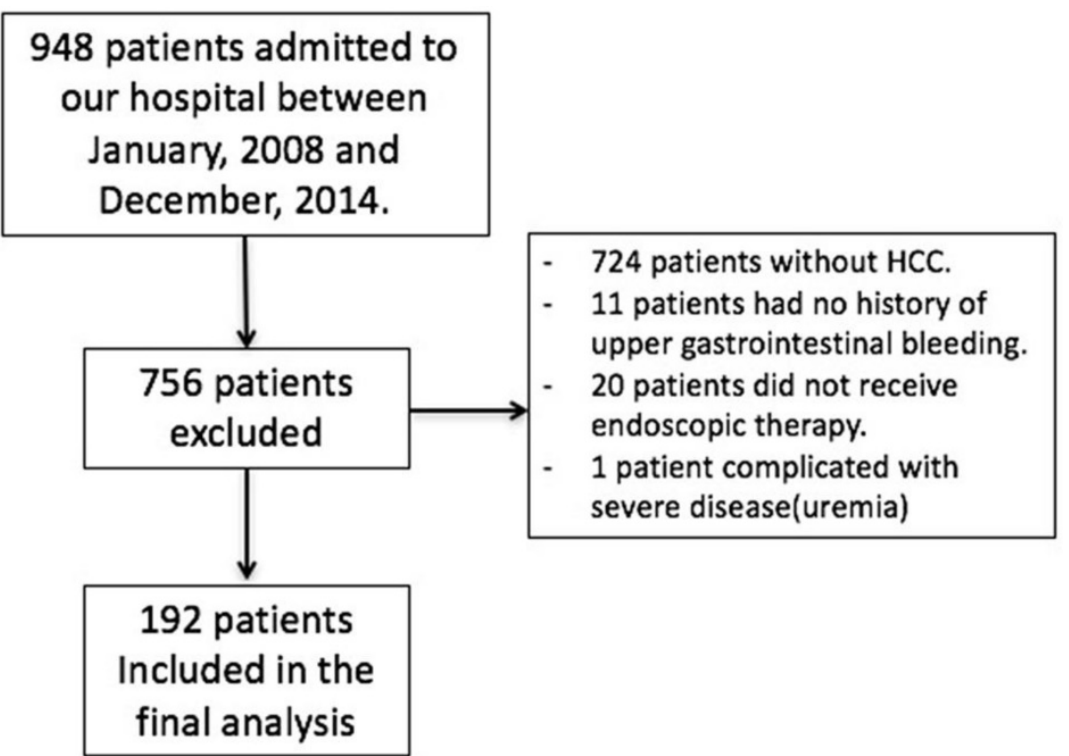

Figure 1. Study flow diagram. The flow diagram showed the inclusion of the patients in our study and gave reasons for non-participation at each stage. 


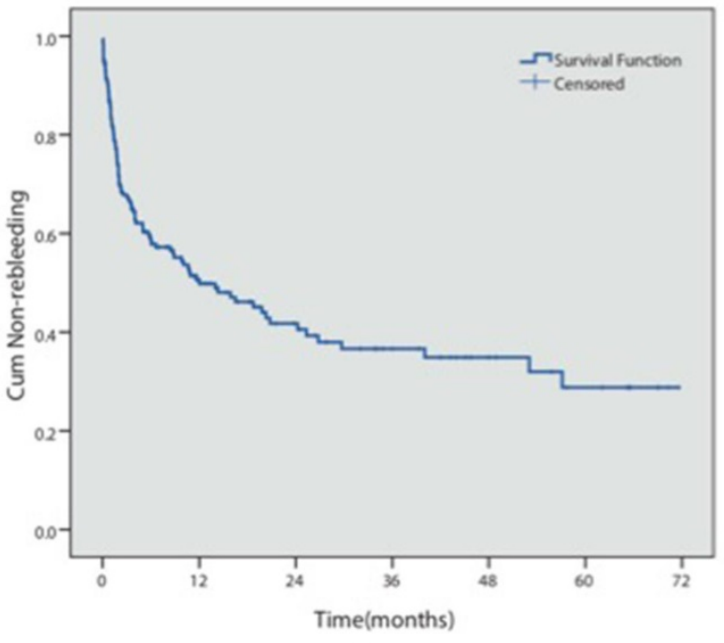

(A)

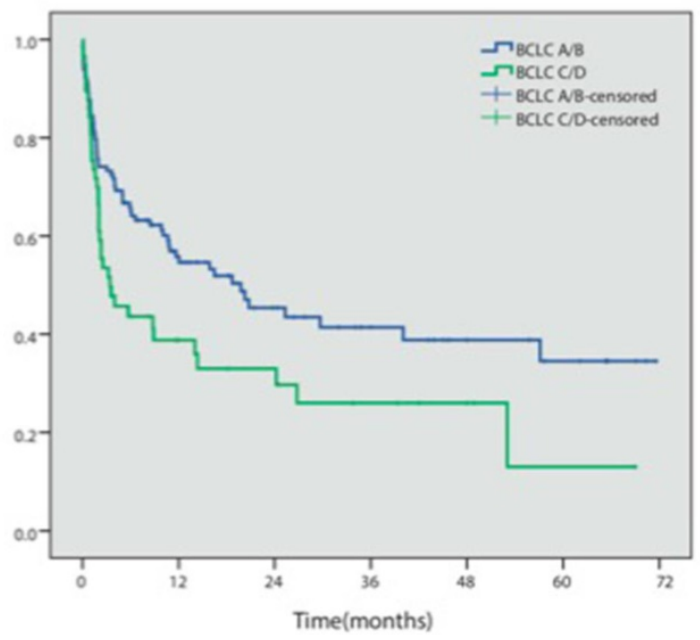

(B)

Figure 2. Rebleeding analysis. (A) The overall cumulative non-rebleeding period in HCC patients complicated with EGV was demonstrated using the Kaplan-Meier survival curve. The cumulative 6 -month, 1 - and 5 -year rebleeding rates were $40.9 \%, 49.3 \%$ and $71.2 \%$, respectively. (B) The cumulative non-rebleeding period in BCLC-A/B and BCLC-C/D patients was compared using the Kaplan-Meier survival curve.

Table 2. Analysis using Cox multivariate proportional hazard models for prognostic factors for re-bleeding and survival

\begin{tabular}{llll}
\hline Analysis & Factor & Multivariate & \\
\cline { 3 - 4 } & & $1.53(1.003-2.327)$ & 0.048 \\
Relapse & BCLC(C/D vs. A/B) & $0.50(0.230-1.077)$ & 0.076 \\
bleeding & Splenectomy(yes vs no) & & \\
& & $1.20(0.797-1.806)$ & 0.384 \\
& Hemoglobin (<90 vs. $\geq 90 \mathrm{~g} / \mathrm{L})$ & $1.58(1.135-2.198)$ & 0.007 \\
& Gender(male vs. female) & $1.59(1.033-2.454)$ & 0.035 \\
& BCLC(C/D vs. A/B) & $0.66(0.379-1.161)$ & 0.151 \\
Survival & Platelet(<90 vs. >90 x1012/L) & $0.68(0.447-1.045)$ & 0.079 \\
& Hemoglobin (<90 vs. $\geq 90 \mathrm{~g} / \mathrm{L})$ & $0.71(0.414-1.227)$ & 0.222 \\
& Diebetes mellitus(yes vs. no) & $0.71(0.393-1.290)$ & 0.263 \\
\hline
\end{tabular}

†BCLC grading: the Barcelona Clinic Liver Cancer grading system

\section{Survival analysis}

A total of 98 patients (51.0\%) died during follow-up, with a median survival time of 18.6 months (see Figure 3 for Kaplan-Meier analysis). Among these patients, 72 patients $(37.5 \%)$ died of GI bleeding. The 6-month, 1- and 5-year overall cumulative survival rates were $66.5 \%, 54.2 \%$ and $34.3 \%$, respectively. During the follow-up period, no major complications or endoscopic therapy-related deaths were observed.

Similar to the re-bleeding analysis, we also conducted univariate and multivariate analyses of the prognostic factors for survival in these patients (Table $2)$. BCLC $C / D$ stage $(P=0.035, H R=1.59,95 \% C I$ 1.033-2.454) and male gender $(\mathrm{P}=0.007, \mathrm{HR}=1.58,95 \%$ CI 1.135-2.198) predicted shorter survival times.

\section{Endoscopic approaches}

Ninety-four patients presented with EV alone, absent of GV. Among them, 83 patients received EVL procedure, among which 45 patients $(54.2 \%)$ had relapse bleeding during follow-up. 11 patients received EIS procedure for EV, among which 6 patients $(54.5 \%)$ had relapse bleeding. Kaplan-Meier analysis indicated that different endoscopic procedures did not influence the risk of rebleeding in patients with EV alone (EVL vs EIS, $\mathrm{P}=0.824$ ).

Eighty-eight patients presented with concomitant EV and GV. Cyanoacrylate injection was conducted for GV in all 88 cases, while EVL was conducted in 70 patients (the EVL group) and EVL combined with EIS was conducted in 18 patients for EV (the EVL+EIS group). During follow-up, 40 patients in the EVL group and 6 patients in the EVL+EIS group had relapse bleeding. 39 cases in the EVL group and 3 cases in the EVL+EIS group died. Kaplan-Meier analysis indicated that the EVL+EIS group presented with lower rebleeding risk $(57.1 \%$ vs $33.3 \%, \mathrm{P}=0.003)$ and better survival $(44.3 \%$ vs $88.3 \%$, $\mathrm{P}=0.005$ ) (Table 3, Fig 4).

Table 3. Rebleeding and death of patients devided by different management for EGV

\begin{tabular}{llll}
\hline Patients(n=182) & Endoscopic procedure & \multicolumn{1}{c}{ Rebleeding } & Death \\
\hline $\begin{array}{l}\text { Esophageal varices alone } \\
(\mathrm{n}=94)\end{array}$ & EVL(n=83) & $45(54.2 \%)$ & $45(54.2 \%)$ \\
& $\begin{array}{l}\text { Combination EVL and EIS(n= } \\
11)\end{array}$ & $6(54.5 \%)$ & $5(45.5 \%)$ \\
$\begin{array}{l}\text { Concomitant esophageal } \\
\text { and gastric varices }(\mathrm{n}=88)\end{array}$ & $\begin{array}{l}\text { EVL + cyanoacrylate injection } \\
(\mathrm{n}=69)\end{array}$ & $38(55.1 \%)$ & $35(50.7 \%)$ \\
& $\begin{array}{l}\text { Combination EVL and EIS + } \\
\text { cyanoacrylate injection(n=19) }\end{array}$ & $7(36.8 \%)$ & $4(21.1 \%)$ \\
\hline †VL: esophageal variceal ligation, EIS: esophageal injection sclerotherapy.
\end{tabular}

\section{Early vs non-early endoscopic treatment}

We defined the early treatment group as initial endoscopic treatment conducted within one week since the bleeding episode, otherwise the non-early treatment group. 78 patients received early endoscopic treatment, among which 47 cases $(60.3 \%)$ 
had relapse bleeding. 55/114 patients $(48.2 \%)$ in the non-early treatment group had relapse rebleeding. Kaplan-Meier curve showed a relatively lower risk of rebleeding within the first 2 years for the non-early treatment group, however not statistically significant $(\mathrm{P}=0.254)$. No further difference was observed between the two groups after 2 years.

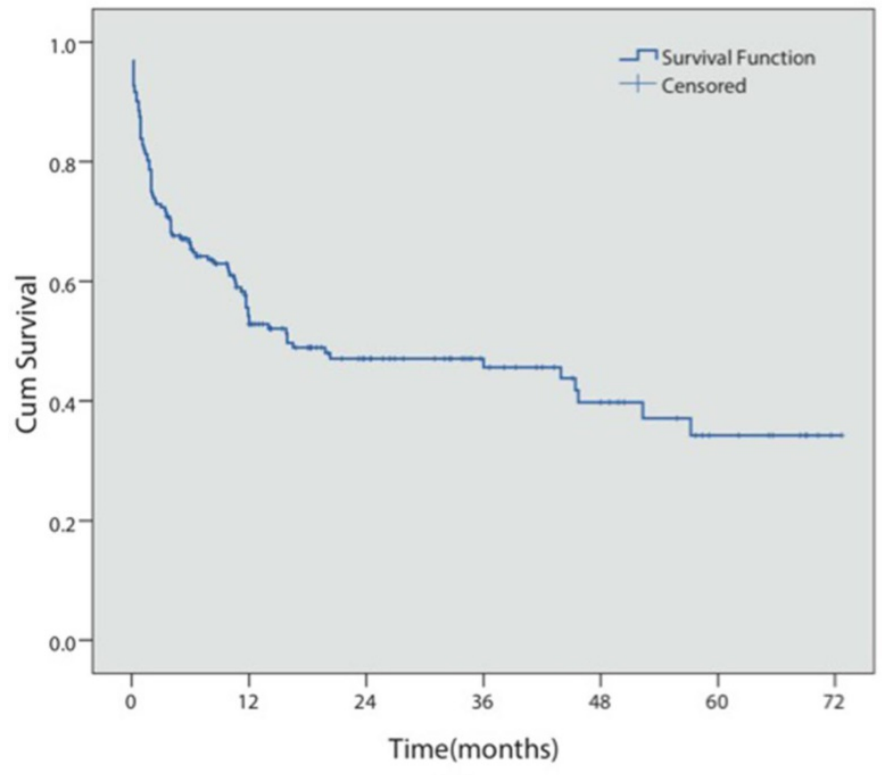

(A)

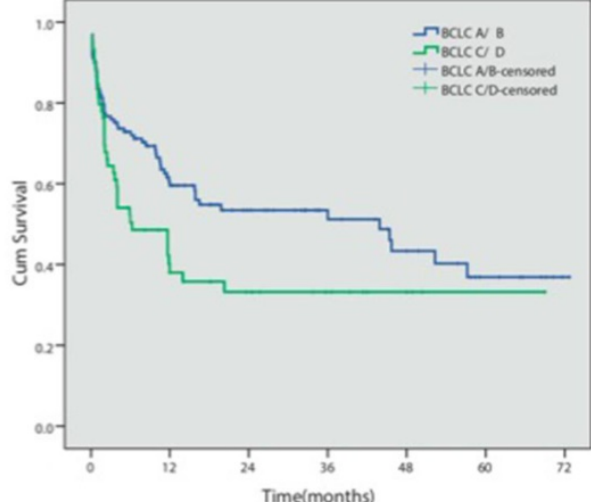

(B)

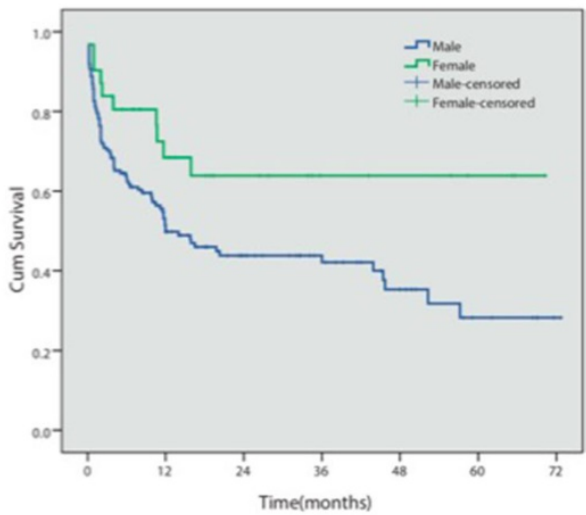

Figure 3.Survival analysis. (A) Overall cumulative survival period in HCC patients complicated with EGV was demonstrated using the Kaplan-Meier survival curve. The cumulative 6 -month, 1 - and 5 -year survival rates were $66.5 \%, 54.2 \%$ and $34.3 \%$, respectively.(B) The cumulative survival period in BCLC-A/B or BCLC-C/D patients was compared using the Kaplan-Meier survival curve. (C) The cumulative survival period in female or male patients was compared using the Kaplan-Meier survival curve.

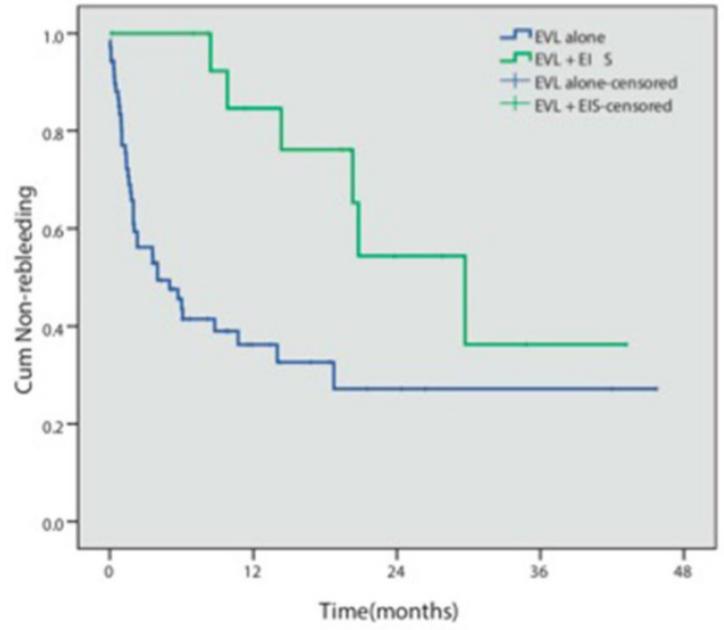

(A)

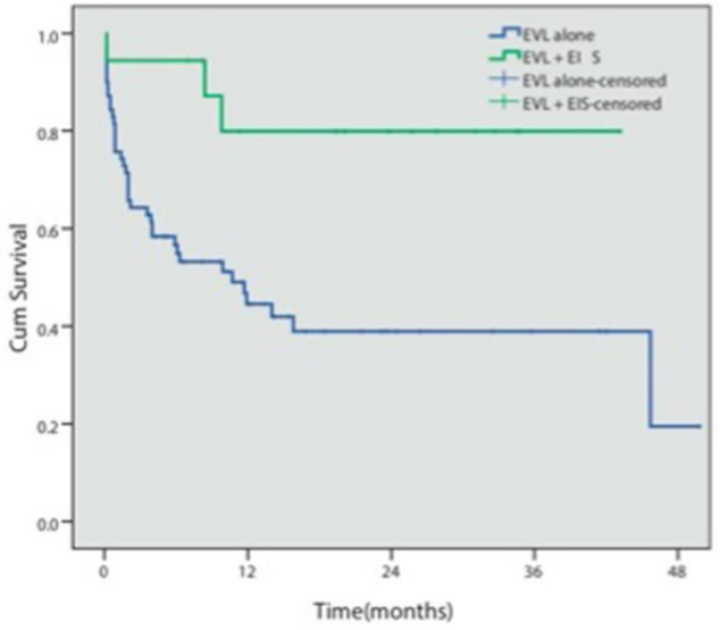

(B)

Figure 4. Different endoscopic approaches. (A) The cumulative non-rebleeding period in the EVL alone group or in the EVL+EIS group was compared using the Kaplan-Meier survival curve. (B) The cumulative survival period in the EVL alone group or in the EVL+EIS group was compared using the Kaplan-Meier survival curve. 


\section{Discussion}

Most patients with HCC have advanced liver cirrhosis and portal hypertension. Tumor infiltration could cause high frequency portal vein thrombosis, contributing to an increase in portal venous pressure. EGV rupture could aggravate hepatic function or because bleeding associated with death and other lethal complications. The actual incidence of HCC, presenting with variceal bleeding, remains unknown, although incidences ranging from $1 \%$ to $15 \%$ have been reported ${ }^{11}$.

As shown in some studies, HCC patients with variceal bleeding had an in-hospital mortality rate of $20.5 \%$.These patients were expected to have a significantly worse survival outcome than general HCC patients (median 3.5 months vs. 7.5 months), suggesting that the presence of EGV greatly impacted survival and could potentially cause bleeding associated with death, even before HCC deterioration ${ }^{12,13}$. Endoscopic treatments as secondary prophylaxis in EGV have long been recognized ${ }^{14}$, but the effects of these treatments on HCC patients have been seldom studied. Keiji $M$, et al. reported that prophylactic sclerotherapy for $\mathrm{EV}$ prolonged the long-term survival of HCC patients, but had no beneficial effects on patients with poor disease status $^{15}$. In a recent study that evaluated outcomes in HCC patients after rebleeding prophylaxis, Ripoll et al stated that even though secondary prophylaxis failure was more frequent and survival significantly shorter in patients with HCC, secondary prophylaxis still offered survival benefit. Lack of prophylaxis increased rebleeding and mortality ${ }^{16}$. Overall, few studies have focused on this specific group of patients.

In recent years, several models have been proposed to predict survival in patients with HCC, including the Italian score (CLIP), the French classification $(\mathrm{GRETCH})$ and the Barcelona (BCLC) staging system 17. Although the overall predictive ability of these systems was not satisfactory, the BCLC staging system has been used as a reference model, as this system associates staging with treatment modalities and the estimation of life expectancy. Consistently in our research, despite that portal hypertension potentially affected the prognosis, the BCLC staging system was still the independent predictor of survival in this subgroup of HCC patients. Furthermore, BCLC staging system showed significant effects on the prediction of rebleeding risk, reflecting the similar demographic and clinical characteristics of our patients compared with general HCC patients. Male gender is associated with poor prognosis, which is consistent with previous studies. In comparison, female patients tend to have more favorable pathological feature of HCC upon initial diagnosis and a greater likelihood to undergo curative therapy ${ }^{18,19}$.

Previous studies suggested no superiority of combined EVL and EIS plus cyanoacrylate injection for variceal bleeding in cirrhotic patients. However, we included certain subgroup of patients with $\mathrm{HCC}^{20}$, 21. In the present study, different endoscopic procedures did not influence the risk of rebleeding in patients with EV alone. Nevertheless, in those with both EV and GV, ligation combined with sclerotherapy plus cyanoacrylate injection was associated with a decreased rebleeding and mortality rate, which suggested combination therapy should be preferred for those patients. Emergent endoscopic therapy has also been shown to be less superior than delayed procedure, in terms of patient outcome and rebleeding rate. Stabilization with pharmacological therapy may be more suitable upon an acute variceal bleeding episode 22,23 .

The study had several limitations. In our study, 7 patients $(3.6 \%)$ were lost to follow up despite our best effort, which could impair the integrality of data and thus influence the validity of the study. Besides, since it was a retrospective study, unmeasured or residual confounding like memory bias might be present. Since it was an observational study and no intervention was involved, many patients did not receive gastroscopy screen after relapse of bleeding. Thus, nor the exact cause of rebleeding either the rate of variceal related bleeding or death could be further confirmed. Last but not least, there were only 2 Child-C patients $(1.0 \%)$ in our study, so it would be cautious to extend our results only in patients with rather reserved liver function. Although age was not a significant factor for rebleeding and survival in the present study, elderly patients are often associated with poor prognosis. Thus, it is important to consider age and other comorbidities when assessing disease prognosis.

In conclusion, the present study demonstrated that for HCC patients complicated by variceal bleeding, combination ligation and sclerotherapy could be preferred for those with concomitant EV and GV. BCLC C/D stage predicted shorter survival times and bleeding relapse, while male gender was independent predictors of poor survival. Start time of the initial endoscopic procedure did not affect efficacy.

\section{Abbreviations}

BCLC: Barcelona Clinic Liver Cancer; HR: Hazard Ratio; HCC: Hepatocellular Carcinoma; EGV: esophagogastric varices; TIPS: Transjugular 
intrahepatic portosystemic shunt; AFP: alpha-fetoprotein; CT: computed tomography; MRI: magnetic resonance imaging; TACE: Transcatheter arterial chemoembolization; RFA: radiofrequency ablation; PEI: percutaneous ethanol injection; EV: esophageal varices; GV: gastric varices; GOV: gastroesophageal varices; EIS: endoscopic injection sclerotherapy; EVL: endoscopic variceal ligation; NBCA: N-butyl-cyanoacrylate; GI: Gastrointestinal.

\section{Acknowledgements}

Professor Shiyao Chen has access to the data and control of the decision to publish. He is accepting full responsibility for the conduct of the study. The authors thank the members of Professor Shiyao Chen's laboratory team for their participation in the discussions to this work.

\section{Source of funding}

The present study was partly supported by the Innovation Fund of Shanghai Scientific Committee (15411950501) and the Science Fund for Young Scholars of Zhongshan Hospital (2016ZSQN11).

\section{Competing Interests}

The authors have declared that no competing interest exists.

\section{References}

1. Sugimoto N, Watanabe K, Watanabe K, et al. Endoscopic hemostasis for bleeding gastric varices treated by combination of variceal ligation and sclerotherapy with N-butyl-2-cyanoacrylate. J Gastroenterol. 2007;42: 528-32.

2. Rockey DC. Screening for esophageal varices in patients with hepatocellular carcinoma. Gastrointest Endosc. 2018;88: 240-1.

3. Berzigotti A, Reig M, Abraldes JG, et al. Portal hypertension and the outcome of surgery for hepatocellular carcinoma in compensated cirrhosis: a systematic review and meta-analysis. Hepatology. 2015;61: 526-36.

4. Serste T, Melot C, Francoz C, et al. Deleterious effects of beta-blockers on survival in patients with cirrhosis and refractory ascites. Hepatology. 2010;52: 1017-22.

5. Chen WC, Hou MC, Lin HC, et al. Feasibility and potential benefit of maintenance endoscopic variceal ligation in patients with unresectable hepatocellular carcinoma and acute esophageal variceal hemorrhage: a controlled trial. Gastrointest Endosc. 2001;54: 18-23.

6. Yang JD, Kim WR, Park KW, et al. Model to estimate survival in ambulatory patients with hepatocellular carcinoma. Hepatology. 2012;56: 614-21.

7. Soga K, Tomikashi K, Miyawaki K, et al. MELD score, child-pugh score, and decreased albumin as risk factors for gastric variceal bleeding. Hepatogastroenterology. 2009;56: 1552-6.

8. CSoHaCSoE CSOG. Consenus on prevention and treatment for gastroesophageal varices and variceal hemorrhage in liver cirrhosis. Chinese Journal of Hepatology. 2008;8: 10.

9. Sarin SK, Kumar A. Gastric varices: profile, classification, and management. Am J Gastroenterol. 1989;84: 1244-9.

10. Sonomura T, Sato M, Kishi K, et al. Balloon-occluded retrograde transvenous obliteration for gastric varices: a feasibility study. Cardiovasc Intervent Radiol. 1998;21: 27-30.

11. Nanashima A, Sumida $\mathrm{Y}, \mathrm{Abo} \mathrm{T}$, et al. Treatment of concomitant gastric varices in patients with hepatocellular carcinoma at a single Japanese institute. Hepatogastroenterology. 2009;56: 857-60.

12. Lang BH, Poon RT, Fan ST, et al. Outcomes of patients with hepatocellular carcinoma presenting with variceal bleeding. Am J Gastroenterol. 2004;99: 2158-65.

13. Iavarone $\mathrm{M}$, Primignani $\mathrm{M}$, Vavassori $\mathrm{S}$, et al. Determinants of esophageal varices bleeding in patients with advanced hepatocellular carcinoma treated with sorafenib. United European Gastroenterol J. 2016;4: 363-70.

14. de Franchis R. Revising consensus in portal hypertension: report of the Baveno $\mathrm{V}$ consensus workshop on methodology of diagnosis and therapy in portal hypertension. J Hepatol. 2010;53: 762-8.
15. Orloff MJ, Isenberg JI, Wheeler HO, et al. A randomized controlled trial of emergency treatment of bleeding esophageal varices in cirrhosis for hepatocellular carcinoma. Am J Surg. 2012;203: 182-90.

16. Ripoll C, Genesca J, Araujo IK, et al. Rebleeding prophylaxis improves outcomes in patients with hepatocellular carcinoma. A multicenter case-control study. Hepatology. 2013;58: 2079-88.

17. Bertuccio P, Turati $\mathrm{F}$, Carioli $\mathrm{G}$, et al. Global trends and predictions in hepatocellular carcinoma mortality. J Hepatol. 2017;67: 302-9.

18. Tangkijvanich $P$, Mahachai V, Suwangool $P$, et al. Gender difference in clinicopathologic features and survival of patients with hepatocellular carcinoma. World J Gastroenterol. 2004;10: 1547-50.

19. Njei B, Rotman Y, Ditah I, et al. Emerging trends in hepatocellular carcinoma incidence and mortality. Hepatology. 2015;61: 191-9.

20. Chen J, Zeng XQ, Ma LL, et al. Long-term efficacy of endoscopic ligation plus cyanoacrylate injection with or without sclerotherapy for variceal bleeding. J Dig Dis. 2016;17: 252-9.

21. Chen J, Zeng XQ, Ma LL, et al. Randomized controlled trial comparing endoscopic ligation with or without sclerotherapy for secondary prophylaxis of variceal bleeding. Eur J Gastroenterol Hepatol. 2016;28: 95-100.

22. Yan BM, Lee SS. Emergency management of bleeding esophageal varices: drugs, bands or sleep? Can J Gastroenterol. 2006;20: 165-70.

23. Tapper EB, Ezaz G, Patwardhan V, et al. Hospital-level balloon tamponade use is associated with increased mortality for all patients presenting with acute variceal haemorrhage. Liver Int. 2018;38: 477-83. 